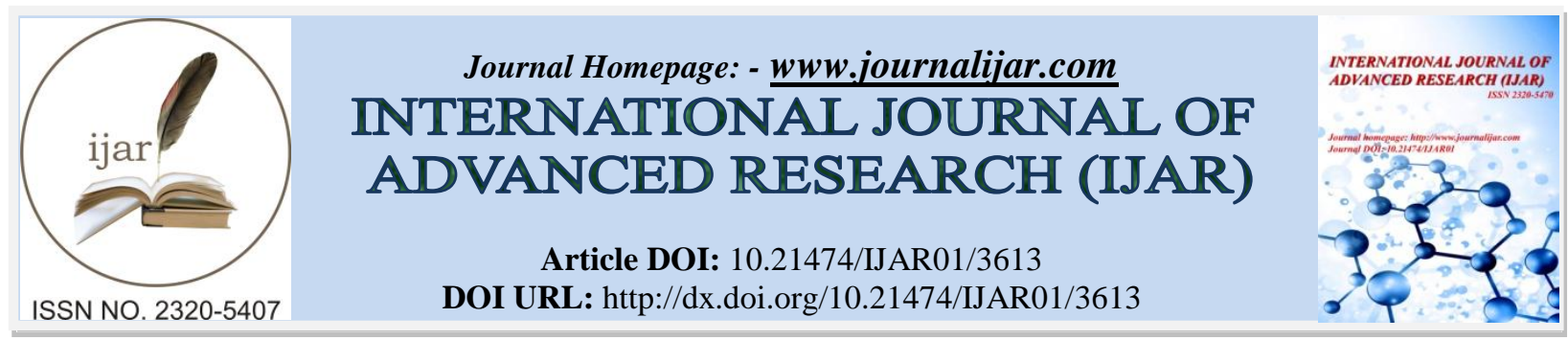

RESEARCH ARTICLE

\title{
CRYOGELS POREUX A BASE DES FIBRES DE LIN TRAITEES PAR UN MELANGE LIQUIDE IONIQUE EMIMAC / DMSO.
}

\section{Rabiaa Hajji*1,2 and Et Ammar Ben Brahim1.}

1. Thermo dynamique Appliquée, Ecole Nationale d'ingenieurs de Gabes, Universite de gabes, Tunisie Avenue Omar ibn el khat tab, zrig eddakhlania, gabes 6029.

2. Centre de Mise en Forme des Materiaux, UMR CNRS 7635, France, Universite de Sophia Antipolis, Nice, France 06904, Sophia Antipolis, Nice, France.

\section{Manuscript Info}

(.........................

Manuscript History

Received: 07 January 2017

Final Accepted: 12 February 2017

Published: March 2017

Key words:-

Biomasse lignocellulosiques, Fibre de

Lin, liquide ionique, MEB, IR ATR-

FTIR, matériaux poreux.

\section{Abstract}

Les cryogels lignocellulosiques ultra poreux sont reconnus comme des matériaux fonctionnels, en raison de ses différences qualitatives dans les propriétés, la densité et la porosité, et diverses compositions chimiques. Les fibres de lin sont très prometteuses car elles présentent une résistance mécanique élevée, une stabilité chimique, une biodégradabilité, et une non-toxicité sont constituées principalement par de cellulose $(72 \%)$. Les cryogels lignocellulosiques ultraléger et très poreux sont préparés par dissolution des fibres de lin dans un mélange de solvant à base de 1-éthyle-3 méthylimidazolium (EMIMAc) et de Diméhylsulfoxide (DMSO). Le biomatériau à base de lin brute présente des propriétés texturales, morphologiques et cristallines remarquables. La dissolution des fibres de lin dans l'EMIMAC / DMSO est partielle, par contre, sa dissolution dans les mêmes paramètres avec de l'EMIMAc pur a été parfaite, et aucun trace des fibres observée par microscopie optique et image morphologique.

Copy Right, IJAR, 2017,. All rights reserved.

\section{Introduction:-}

Les politiques actuelles de synthèse des matériaux se basent sur trois grands principes: le respect de l'environnement et des hommes, la réduction des déchets et des matières polluantes et le remplacement partiel ou total des dérivés pétrochimiques par des ressources renouvelables lignocellulosiques. Ainsi, la valorisation de la biomasse lignocellulosiques est favorisée par deux facteurs. D'une part, l'industrie des matières plastiques doit faire face à une diminution des dérivées pétrochimiques, d'autre part à une prise de conscience grandissante des populations de l'impact des matériaux plastiques sur notre environnement (dégagement de CO2). Alors des stratégies connues pour convertir la lignocellulose en matériaux poreux impliquant différentes étapes d'élaborations suivies par des traitements chimiques sont recommandé. Parmi ces stratégies nous avons pensé à la solution suivante : La synthèse des matériaux poreux sans recours à des traitements chimiques.

C'est pour cela que nous avons pensé à une matière lignocellulosiques brute qui sera traité par le procédé que nous allons décrire. L'utilisation des matières primaires lignocellulosique pour la fabrication de produits respectueux de l'environnement a généré beaucoup d'études, avec différentes étapes nécessaires pour l'obtention d'un produit tel que

\section{Corresponding Author:- Rabiaa Hajji.}

Address:- Thermo dynamique Appliquée, Ecole Nationale d'ingenieurs de Gabes, Universite de gabes,

Tunisie Avenue Omar ibn el khat tab, zrig eddakhlania, gabes 6029. 
Tous les axes de recherche sont d'une grande importance dans le domaine de la lignocellulose. Cependant, une attention particulière a été donnée aux aspects d'accessibilité de la Fibre de lin. Il est à noté que cette fibres est mondialement connue, elle est utilisé actuellement pour la fabrication des plusieurs bio composites (les chaises, les cadres de lunette...).

D'autant plus, beaucoup des procèdes chimiques qui sont utilisés pour séparer la cellulose et l'hémicellulose de la biomasse lignocellulosique, car la lignine molécule très solide, sont à la fois lourds et coûteux. Nous tenons à préciser qu'aucun travail n'a été effectué dans ce domaine en utilisant la dissolution des fibres de lin brute dans l'EMIMAc/DMSO.

Dans cet article nous résumons les différentes étapes de synthèse et caractérisation que nous avons suivie pour atteindre notre but.

\author{
Etudes experimentales :- \\ Materiels et produits chimiques:- \\ Nous avons utilises:- \\ $>$ Des fibres de lin de longueur $0.5 \mathrm{~mm}$ de la part de Dehondt-France, \\ $>$ Un liquide ionique : 1-Éthyle-3-méthylimidazolium acétate 97\% (EMIMAc, Sigma-Aldrich), \\ $>$ Un co-solvant : Diméthyle sulfoxide (DMSO), \\ $>$ Des coagulant non solvant : de l'eau dé-ionisée et de l'acétone absolue \\ $>$ Tous les réactifs ont été utilisés tels que reçus sans aucune purification.
}

\title{
Procedes De Préparations Des Solutions Lin/EMIMAc-DMSO (80-20):- Etapes de prétraitement et dissolution des fibres de lin:-
}

Nous avons séché les fibres de lin avant dissolution à une température de $50^{\circ} \mathrm{C}$ sous vide pendant 2 heures, à fin d'éliminer tous trace d'eau adsorbé. Puis ces fibres ont été dissoutes dans un mélange EMIMAc/DMSO (80/20) en poids à $70^{\circ} \mathrm{C}$ pendant environ 124 heures sous agitation magnétique de $500 \mathrm{rpm}$. Les rapports des fibres de lin: solvant mixte étaient 1:99, 2:98 et 3:97 (\% masse / masse). Les solutions ont été respectivement marquées comme FL1, FL2 et FL3.

Enfin, lorsque des solutions colorées, homogènes et visqueuses ont été obtenues, une centrifugation des solutions obtenues a eu lieu pour bien séparer la phase gel de la phase non dissoute. En fin ces solutions ont été stockées à température ambiante et protégées contre l'absorption d'humidité.

\section{Etapes de Régénération:-}

L'acétone et l'eau dé-ionisée ont été utilisées comme non-solvants pour les bains de régénérations. Les solutions obtenues ont été versées dans des tubes en polypropylène, puis de l'acétone pure y a été ajouté soigneusement. Ce mélange a été mis au repos pendant 6 jours pour favoriser la diffusion solvant co-solvant. Ces solutions prérégénérées obtenues ont été recouvertes de l'acétone périodiquement changée (4 fois/jour) pendant 10 jours. Une étape de changement de non solvant a eu lieu après la régénération des solutions dans l'acétone, dans des tubes en polypropylène remplie d'eau bi-distillées pendant $48 \mathrm{~h}$ à fin de remplacer tous traces d'acétone par celui de l'eau. Durant cette mise en forme la teneur en solvant dans les solutions FLX(x : 1, 2 ou $3 \%$ en Fibres de lin) varie de très important lors de la dissolution, elle passe à très faible lors de la fin de régénération.

\section{Etapes de Séchage: Lyophilisation:-}

Tous les échantillons ont été séchés à 40 mTorr et $-80^{\circ} \mathrm{C}$ pendant $48 \mathrm{~h}$ dans un lyophilisateur (Cryotec Comos 80 ). Après la lyophilisation, on obtient des cryogels de dimensions définies. Les cryogels LIN/EMIMAc-DMSO ont été marqués comme CF01, CF02 et CF03, respectivement.

\section{Caractérisations:-}

Des observations microscopiques ont été effectuées après avoir placé les fibres de lin en contact avec le solvant mixte entre deux lames de verre, par un microscope optique métallographique inversé (Olympus PMG3) de hautes résolutions. Les photos d'agrandissement 500X ont été prises par un logiciel Archimed.

Aussi les mesures rhéologiques en écoulements des solutions de Fibre de lin/EMIMAc-DMSO ont été effectuées sur un rhéomètre Bohlim Gemini équipé d'une géométrie cone-plan $\left(4^{\circ}-40 \mathrm{~mm}\right)$ et d'une platine Peltier pour le contrôle 
de la température. La dépendance de la viscosité en fonction du taux de cisaillement pour les solutions de cellulose (Lin)/EMIMAc a été enregistrée à températures fixes de 0 a $100^{\circ} \mathrm{C}$, par incrément de $5^{\circ} \mathrm{C}$.

Pour savoir si ces travaux ont eux des résultats nous avons procédé par une spectroscopie infrarouge à transformée de Fourier (ATR-FTIR, Spectrum Two Perkin Elmer), pour étudier la composition initiale des fibres et les cryogels. Le traitement spectroscopique a été enregistré dans la gamme de longueur d'ondes de 4000-500 $\mathrm{cm}^{-1}$. Les échantillons ont été balayés 10 fois avec une résolution spectrale de $4 \mathrm{~cm}^{-1}$.

D'autre par, la morphologie des échantillons métallisés a été examinée au microscope électronique à balayage (SEM Zeiss Supra 40 FEG) à une tension d'accélération de $3 \mathrm{kV}$.

Tandis qu'aux les surfaces spécifiques des Cryogels (SBET) ont été calculées selon la théorie de Brunauer-EmmettTeller (BET), en utilisant des isothermes d'adsorption-désorption d'azote (ASAP 2020, Micromeritics) à $-196{ }^{\circ} \mathrm{C}$.

Les densités apparentes des échantillons ont été mesurées en utilisant le pycnomètre à poudre Geopyc 1360 de Micromeritics.

La synthèse ainsi que les caractérisations des échantillons ont été étudiées au centre de mise en forme des matériaux, l'école supérieur des mines de Paris, Sophia Antipolis-Nice, France.

\section{Résultats Et Discussion :-}

Gonflement et dissolution des fibres de lin : vue microscopique:-

Dés le début et par différents grossissement nous avons remarqué que le système lin/solvant a provoqué un gonflement des fibres de lin. Plus précisément avec l'analyse microscopique, il s'est avéré que ce gonflement est en réalité un ballonnement sous forme de chapeler de petit ballon (Fig.1).

En effet les fibres de lin gonflent de façon homogène, le solvant mixte réagit comme agent gonflant. Le mécanisme de gonflement et de dissolution est identique à celui de la cellulose dans les liquides ioniques [4]. Cela indique que le processus de dissolution des fibres de lin brute n'est pas contrôlé uniquement par la nature chimique du solvant mais également par la qualité lignocellulosiques de la matière ( $72 \%$ de cellulose dans le lin).

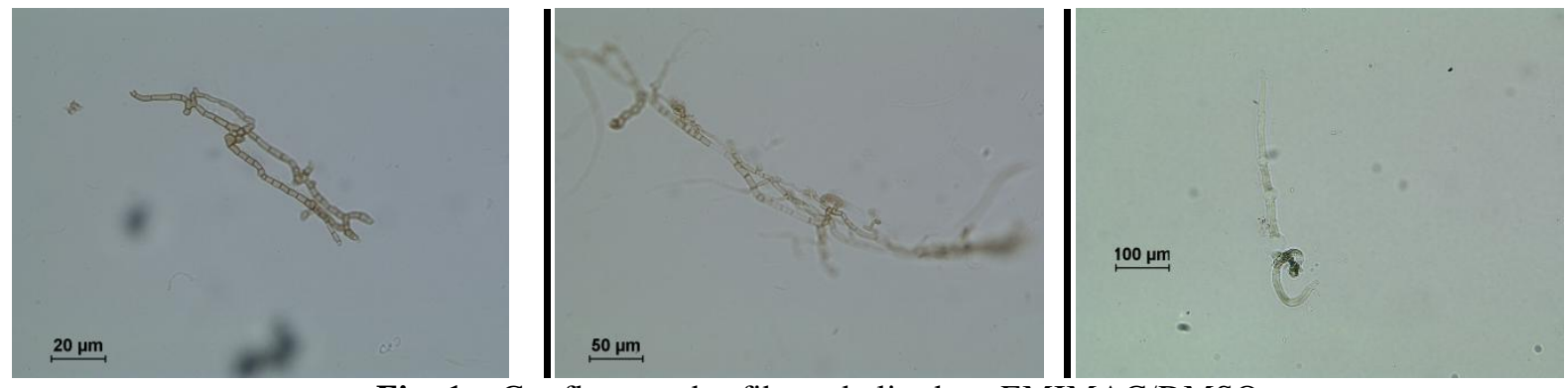

Fig. 1. Gonflement des fibres de lin dans EMIMAC/DMSO

Les paramètres clé influençant les phénomènes de gonflement et de dissolution des fibres de lin sont liés à leur composition lignocellulosiques, la structure morphologique et le degré de polymérisation élevés $(\mathrm{Dp}=9000)$ [5]. Après $10 \mathrm{~h}$ de traitement sous agitation magnétique à température égale à $70^{\circ} \mathrm{C}$, les ballons ont commencé a éclaté, pour favoriser la dissolution et la formation d'une matière visqueuse.

Ce prétraitement permet une solubilisation quasi-totale de la lignine (coloration brunâtre des solutions) et des hémicelluloses avec le temps. Le degré de polymérisation $\left(\mathrm{DP}_{\text {lin }}\right)$ et le taux de cristallinité de la cellulose du lin sont aussi diminués. Les fibres de lin, composées majoritairement de la cellulose (72\%) [6], elles prennent majoritairement les mêmes caractéristiques de gonflement que celui de la cellulose. Il a été rapporté que se phénomène a été déjà étudiée pour le coton et les fibres de bois [7], en liquide ionique avec quelques grammes de DMSO suivent la même séquence de dissolution suivant quatre phases :

- Phase 1: Ballonnement,

- Phase 2: Éclatement du ballon, 
- Phase 3: dissolution des sections non ébouriffées (Rupture),

- $\quad$ Phase 4: dissolution des restes de membrane des ballons.

La structure supramoléculaire des polysaccharides de lin est détruite d'une façon irréversible par l'effet des paramètres de dissolution (solvant, température, temps de réaction et agitation).

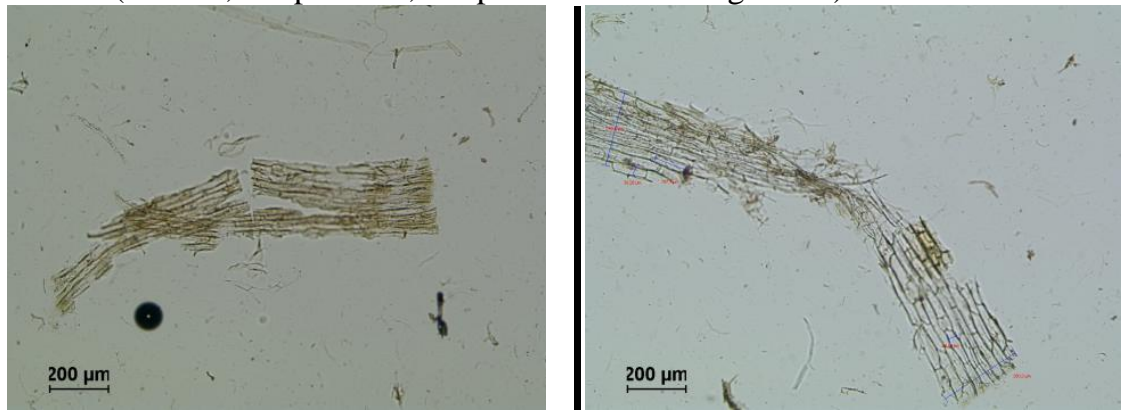

Fig. 2. Rupture des fibres au cours d'agitation

L'intérêt des liquides ioniques dans le cadre de notre étude repose sur leurs remarquables capacités à dissoudre des composés lignocellulosiques ou polymériques brutes, polaires ou non polaires, organiques ou inorganiques. Il a ainsi été montré que certains liquides ioniques pouvaient dissoudre des polysaccharides telles que la chitine [9] et la cellulose [10].

La rupture (Fig.2) n'apparait jamais de façon brutale mais elle se déroule progressivement après que la fibre ait subi des étapes de gonflements successifs. Les fibres se déchirent peu à peu, suite à des efforts d'agitation et de température (Fig.2). La rupture des fibres de lin ne se déroule pas facilement. Elle se manifeste sous l'effet d'un phénomène de fatigue, après une certaine accumulation du cisaillement et des contraintes dans les liaisons internes des composées lignocellulosiques du lin.

Néanmoins, des solutions parfaites des polymères dissous totalement sont rarement observables même macroscopiquement. Ce pendant lorsque nous n'avons pas utilisé le co-solvant « DMSO » nous avons obtenues une dissolution parfaite. Ce qui montre que se co-solvant entrave la perfection de dissolution.

\section{Courbes d'écoulements:-}

Généralement le comportement rhéologique des solutions dépend largement de la nature de la matière qui les constitue (la concentration et de la température). La Figure 4 présente les courbes d'écoulement des solutions de lin qui se comportent comme des gels très visqueux après l'étape de dissolution.

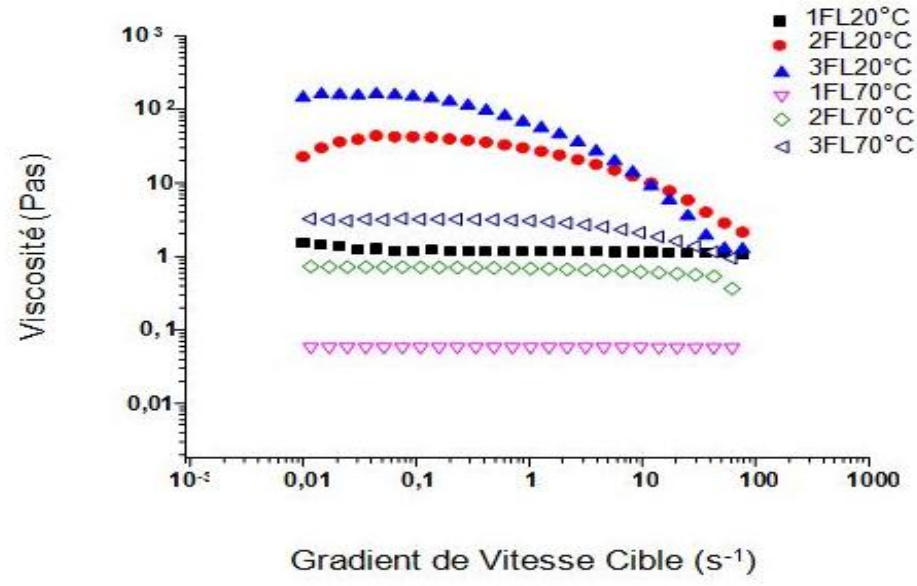

Fig. 3. Courbes d'écoulement des fibres de lin DANS EMIMAC/DMSO.

Il est a noté aussi qu'à concentration et à température faible $\left(1 \%\right.$ massique, $\left.20^{\circ} \mathrm{C}\right)$, nous obtenons des courbes de tendances linéaires (courbe noir), ainsi que pour des valeurs de températures élevés quelques soit le degré de 
concentration. Par contre pour un degré de concentration élevé et une température faibles nous avons obtenues courbes présentent un comportement Newtoniens.

L'augmentation de pourcentage de lin conduit à une augmentation progressive de viscosité. De plus l'influence du DP des fibres de lin sur la viscosité élongationnelle est aussi très importante pour des faibles déformations. Cela corroborait, par plusieurs travaux antérieur, qui ont montré que la viscosité est très importante [11], si le DP est plus élevé (degré de polymérisation de lin égale a 9000) [5].

\section{Caractérisations texturales des cryogels:-}

La lyophilisation produit des cryogels hautement poreux et à faible densité mais à un effet destructeur sur la structure du réseau sur tout pour les concentrations massique faibles (pour une concentration massique de $0,5 \mathrm{~g}$ en fibres de lin l'échantillon sa tient pas malgré la dissolution totale du lin).

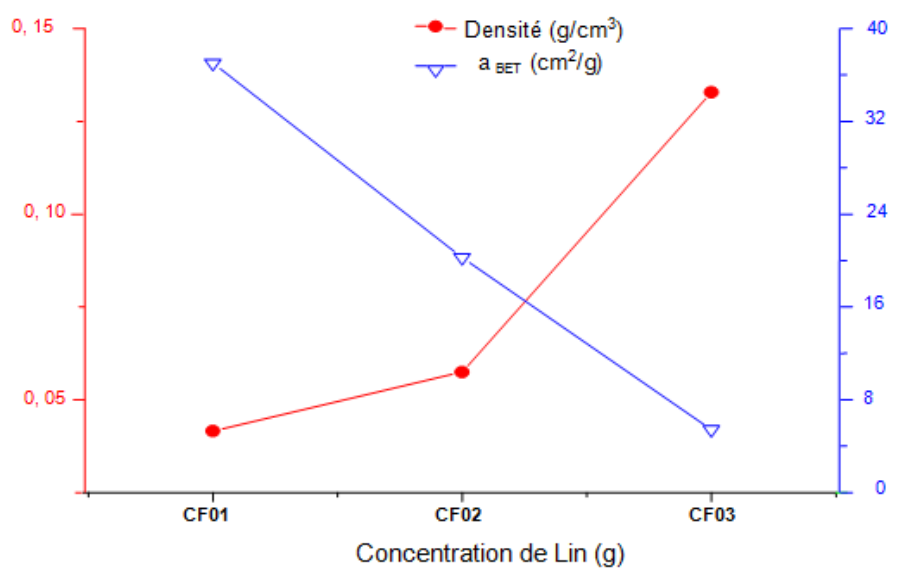

Fig. 4. Courbes de densité et de surface spécifique des cryogels en fonction de la concentration massique de fibre de lin.

L'augmentation de la concentration en fibres de lin à toujours pour conséquence la densification du réseau (Fig.5), et une diminution de porosité et de la surface spécifique des cryogels de lin. La porosité des cryogels synthétisées a été aussi déduite par les images morphologiques.

\section{Morphologies des cryogels de lin:-}

La morphologie des échantillons lyophilisés a été examinée par MEB (Fig.6). Les échantillons cryogels ont été étudiés en morphologie à l'intérieur et à la face supérieure pour les trois concentrations déjà mentionné.
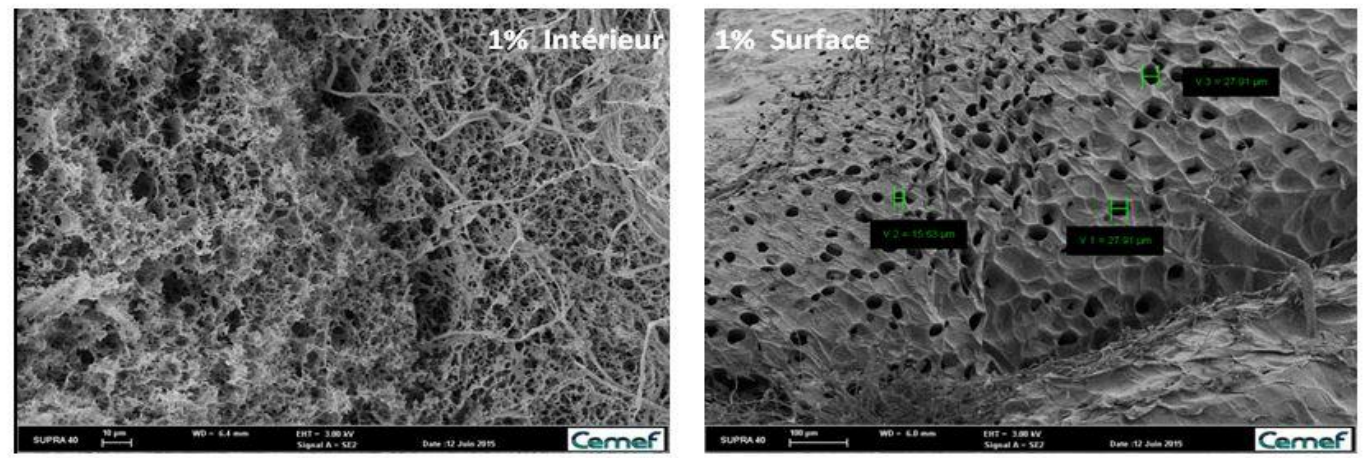

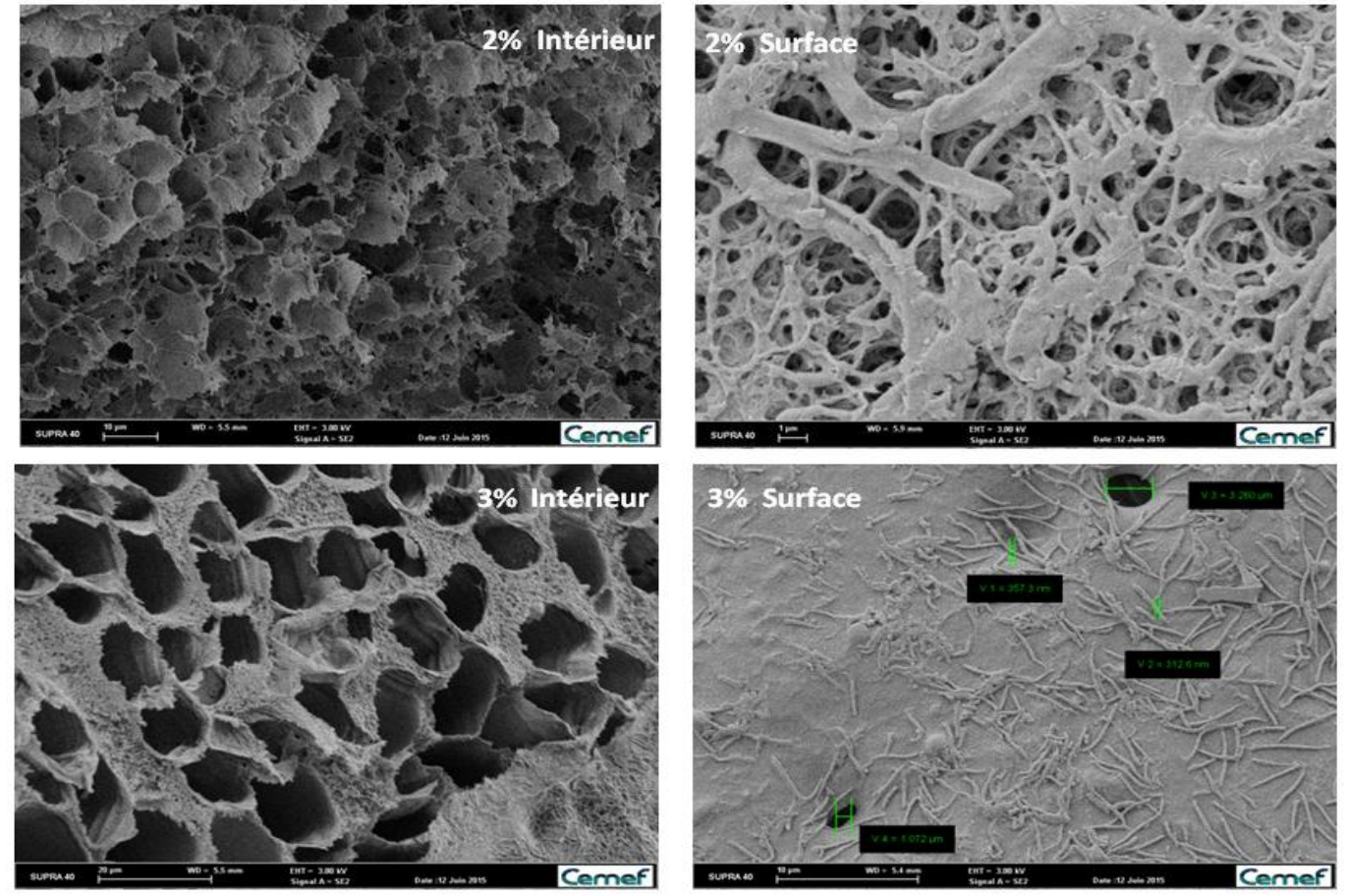

Fig. 6:- Les images morphologiques de coupes intérieures et la surface supérieur des cryogels CF01, CF02 et CF03.

Les faibles grossissements permettent de discerner un réseau poreux à l'intérieur et un réseau solides avec apparition des traces des fibres de lin non dissoutes à la surface supérieur des cryogels (Fig.6). A ce niveau de grossissement, on peut distinguer la haute porosité des cryogels élaborées ainsi que les zones plus foncées (noir), qui doivent correspondre à du vide, donc vraisemblablement à un réseau poreux des matériaux.

La formation du réseau poreux apparait pour les différentes concentrations des fibres de lin. On peut distinguer que pour une concentration égale à $1 \%$ en masse de lin $(1: 99)$ le réseau n'a aucune structure ordonnée (zone feuilleté et zone fibrillaires), par contre pour celui de $2 \%$ en masse un réseau poreux se présente sous forme des feuilles, attachés les uns aux autres. Le réseau poreux feuilleté se densifie avec l'augmentation de la concentration pour former un réseau poreux de structure ordonné en nid d'abeilles de parois poreux

On peut déduire que les parois de cryogel CF1 sont poreuses et fibreux. De ce faite la porosité des cryogels apparait à différentes échelles de micro à nano-pores. La densification des cryogels avec l'augmentation en tenure de lin a été déjà mentionnée (Fig.5), et les images morphologiques confirme cette densification avec la diminution progressive de porosité (nombre des pores sur la surfaces de cryogels 3\%) par rapport à celui de $1 \%$ en lin. L'apparition des traces de fibres de lin pour les cryogels synthétisées à différentes concentrations exprime que le type de solvant EMIMAC/DMSO n'est pas un bon solvant pour le lin. Cette dissolution partielle est influe par l'ajout de co-solvant diméthyle-sulfoxide.

\section{Études spectrales des cryogels ATR-FTIR:-}

Les spectres infrarouges obtenus pour les fibres de lin et le cryogel de lin sont présentés sur la figure 7. Le but de cette partie est de savoir la différence entre la matière primaire brute et le cryogel synthétisée à partir des fibres de lin par spectroscopie infrarouge. 


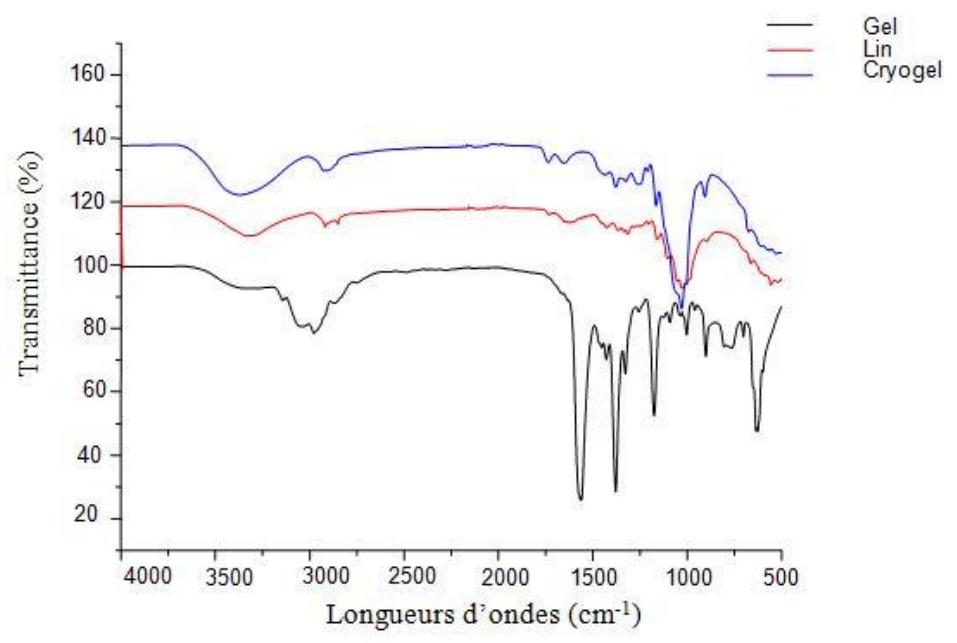

Fig.7. Les Spectres infrarouge de lin brute, solution lin/solvant et cryogel élaborées.

La comparaison des deux états de synthèses (fibres de lin brute, gel de Lin-Solvant) avant le séchage par lyophilisation confrontées afin d'évaluer l'effet de solvant sur le comportement structurale des fibres de lin.

L'apparition d'un enchevêtrement dans l'intervalle 1750-500 $\mathrm{cm}^{-1}$ pour les gels de lin en comparaison avec le spectre des fibres de lin brute. Cette enchevêtrement présente les pics caractéristiques de liquide ioniques, et due à la présence des traces de lignine, hémicelluloses et des extractibles dans la solution lin-solvant, qui disparu avec le changement successive de bain de régénération (coloration foncé du premier bain exprime la présence de forte quantité de lignine soluble dans les gels, cette coloration devient claire avec le changement successifs des bains de régénération jusqu'à ce que le bain sera incolore : couleur de coagulant). Il est toute fois possible de distinguer le pic de la ligne de base aux alentours de $1730 \mathrm{~cm}-1$ [12].

Cette intensification des pics de cryogels dans les deux intervalles $1000-2000 \mathrm{~cm}^{-1}$ et $2750-3500 \mathrm{~cm}^{-1}$ par rapport à celui de spectre du lin brute peut être interpréter par l'importance de la méthode de séchage sur la structure spectrale finale de cryogel. On peut déduire que le spectre infrarouge de cryogel possède des intensités des pics plus importants par rapport aux fibres de lin brut. Cette intensification peut être retenue par la formation des cristaux de glace le long de l'étape de sublimation qui influe sur la structure interne du matériau et sur l'ordre latérale des matériaux (formation d'un réseau ordonné de nid d'abeille (Fig.6).

\section{Conclusion:-}

Les premiers échantillons élaborés dans le cadre de ce travail ont été considérés comme des matériaux de haute porosité à base de fibre de lin brute. Néanmoins la dissolution est partielle, le pourcentage de dissolution est de l'ordre de $85 \%$ de grandeur de celle des fibres de lin dissoute classiquement dans l'EMIMAc pur.

Les observations effectuées à l'aide de la microscopie optique montrent des importantes étapes de changement de la morphologie des fibres lors de l'étape de dissolution (des fibres gonflé par ballonnement, éclatement des ballons, dissolution des membranes externes des ballons...). Le gonflement du lin est observé au premier $6 \mathrm{~h}$ de dissolution, mais après les 6 heures le gonflement disparait avec le temps (éclatement des ballons), qui correspondent également à la dissolution progressive des fibres de lin.

La microscopie électronique à balayage a permis de compléter les informations obtenues par microscopie optique, sur la dissolution partielle des fibres de lin, par l'apparition des traces des fibres de lin non dissout à l'intérieur et la surface des cryogels. En effet, ce que l'on a pu observer c'est un trace des fibres de lin non dissolu avec diminution de leurs longueurs et largeur initiale cela est à cause du fort degré de polymérisation de lin $\left(\mathrm{DP}_{\text {lin }}=9000\right)$ et l'effet de l'ajout de DMSO.

Il semble que la structure cristalline soit plus affectée à une plus grande concentration en lin. 
Les résultats préliminaires de notre étude sur les moyens d'augmenter l'accessibilité de convertir les fibres de lin en matériaux hautement poreux. Des analyses plus détaillées doivent être effectuées. Par exemple, il serait intéressant de dissoudre la fibre de lin brute avec d'autres types de solvant plus efficaces pour minimiser le temps de traitement et le pourcentage de dissolution.

\section{Remerciements:-}

Je tiens à remercier ma directrice de recherche, Tatiana Budtova pour son encadrement et ses encouragements depuis mon stage de recherche. Merci Tania du fond du cœur pour tes bons conseils, ton professionnalisme mais aussi pour ton cote très chaleureux et ton rire expressif qui égaye encore les couloirs du labo !

\section{References:-}

1. Sun, Y. and J. Cheng: Hydrolysis of lignocellulosic materials for ethanol production: A review. Bioresource Technology 83(1): 1-11, 2002.

2. Zaldivar J., Nielsen J. \& Olsson L., Fuel ethanol production from lignocellulose: a challenge for metabolic engineering and process integration. Appl. Microbiol. Biotechnol., 56, 17-34, 2001.

3. Balanis, Antenna Theory: Analysis and Design, 2nd Ed. Wiley India Pvt. Limited, 2007.

4. M. Mazza. Modification chimique de la cellulose en milieu liquide ionique et $\mathrm{CO}_{2}$ supercritique, 2009.

5. Daneault $\mathrm{C}$, Chimie du bois et des dérivés cellulosiques, CHM-GOOl, UQTR

6. Yan L, Chouw N, Jayaraman K. Flax fibre and its composites - A review. Compos Part B Eng ; 56:296317.Compositesb, 2014.

7. C.Cuissinat, P.Navard, T.Heinze. Swelling and dissolution of cellulose. Part IV: Free floating cotton and wood fibres in ionic liquids. Carbohydrate Polymers, Elsevier, 72 (4), pp.Pages 590-596, 2008.

8. Lee S.H., Lee S.B., The Hildebrand solubility parameters, cohesive energy densities and internal energies of 1alkyl-3-methylimidazolium-based room temperature ionic liquids, Chem. Commun., 3469-341, 2005.

9. Xie H., Zhang S., Li S., Chitin and chitosan dissolved in ionic liquids as reversible sorbents of CO2, Green Chem., 8: 630-633, 2006.

10. R.P Swatloski., S.K. Spear., J.D. Holbrey., R.D. Rogers Dissolution of cellulose with ionic liquids, Journal of the American Chemical Society, 124 (18): 4974-4975, 2002.

11. Petrovan et al.: "Residue Met156 contributes to the labile enzyme conformation of coagulation factor VII a", the journal of biological chemistry, vol. 276, no. 9. 6616 - 6620, XP002909202. 2001.

12. M. Mathieu., Modification chimique de la cellulose en milieu liquide ionique et CO supercritique, 2009. 